\title{
Positive mutations and mutation-dependent Verhulst factor in Penna ageing model
}

\author{
S. Moss de Oliveira ${ }^{1}$, D. Stauffer ${ }^{2}$, P.M.C de Oliveira ${ }^{1}$ and J.S. Sá Martins ${ }^{1}$. \\ Laboratoire PMMH, École Supérieure de Physique et de Chimie Indus- \\ trielles, 10 rue Vauquelin, F-75231 Paris, Euroland \\ 1 Visiting from Instituto de Física, Universidade Federal Fluminense; Av. \\ Litorânea s/n, Boa Viagem, Niterói 24210-340, RJ, Brazil; suzana@if.uff.br, \\ jssm@if.uff.br, pmco@if.uff.br. \\ ${ }^{2}$ Visiting from Institute for Theoretical Physics, Cologne University, D-50923 \\ Köln, Euroland; stauffer@thp.uni-koeln.de
}

Abstract: We twice modify the Penna model for biological ageing. First we introduce back (good) mutations and a memory for them into the model. It allows us to observe an improvement of the species fitness over long time scales as well as punctuated equilibrium. Second we adopt a food/space competition factor that depends on the number of accumulated mutations in the individuals genomes, and get rid of the fixed limiting number of allowed mutations. Besides reproducing the main results of the standard model, we also observe a mortality maximum for the oldest old.

Keywords: Evolution, Monte Carlo Simulation, Ageing, punctuated equilibrium PACS: $87.10+\mathrm{e}, 87.23-\mathrm{a}, 05.65-\mathrm{b}$.

\section{Introduction: the standard Penna model}

For biological ageing, the Penna model 1] presently is the most widespread computer simulation method. The genome of each individual is given by a string of 32 bits, representing dangerous inherited diseases (detrimental mutations) for the at most 32 time intervals during the life of this individual. A 0-bit means health, a 1-bit on position $a$ of the bit-string means a harmful mutation affecting the health from that age $a$ on. Three such diseases kill the individual at that age $a$ at which the third disease becomes active. Besides these deaths from genetic reasons, individuals also die at each time step with the Verhulst probability $N / N_{\max }$ where $N$ is the total population and 
$N_{\max }$ a constant parameter, often called the carrying capacity. At each time step, i.e. one iteration of the whole population, each living individual above a minimum reproduction age of 8 gives birth to children with the same genome as the mother except for one mutation in a randomly selected position. For more results from the Penna model we refer to 2, 3. In particular, this model was used to compare various forms of sexual and asexual reproduction for diploids [4].

\section{Model with good mutations}

We have been often criticized for working only with bad mutations. In this case the bit position randomly selected in the parent's genome has its bit set to one independently of its previous value. So the offspring genome is worse or equal to that of the parent, but never better. How can it then be compatible with the Darwinian evolution of more complex and fitter species? The answer is that the Penna model was invented originally [1] to describe ageing, which happens for homo sapiens within a century, and not to describe the evolution of homo over millions of years after the separation from other primates. On short time scales, nearly all mutations are bad; on long time scales, the rare good mutations dominate Darwinian evolution of fitter species. We now aim to include this effect of two time scales into the Penna model.

We start with the asexual Fortran program published in [2] with one bitstring of 32 bits as standard "genome", and with one child born per surviving adult parent per iteration. When the child is born, a randomly selected bit of the standard genome becomes set (one) if it was zero before; it stays set otherwise. When in a first modification we allowed positive mutations (reversing a set bit to a zero bit) with probability 0.01 , we found improved fitness as shown by a higher population, but no evolution over long times.

In our second modification, we return to the case where only bad mutations are allowed: genetic improvement will be represented now by the possibility of not counting some of them, as follows. Each individual gets a second such bit-string as memory for good mutations. At birth, this memory genome gets, with a low probability $q$, on that same bit position where the standard genome is mutated, a bit set to one if it was zero before (otherwise it stays at one). In the evaluation of the active number of mutations (three of which are lethal) those bit positions are ignored. That is, if an individual has a bit set in a given position of the standard genome and also a bit set 
in the same position of the memory genome, then that mutation is ignored (similar to recessive mutations in sexual reproduction [2, 4]). Now we found the desired long-term evolution, but it approached the unrealistic ideal of no active mutations at all, which also means no ageing. The reason is that if a bit is set in the memory string of the parent, it stays necessarily set in the offspring one.

Only our third modification had the desired effect of allowing long-term improvement but still with ageing. At birth, the above memory genome gets, again on the same position as the mutation in the standard genome, a bit set to zero with probability $q$; if it was already zero it stays at zero. Thereafter, with an even smaller probability $q^{2}$, the same bit is set to one. Then, the two mutated bit-strings, i.e. the standard and the memory genome, are given on to the offspring. Only the standard mutations at positions where the memory bit is zero have detrimental effects. The mutation part of the modified program now is:

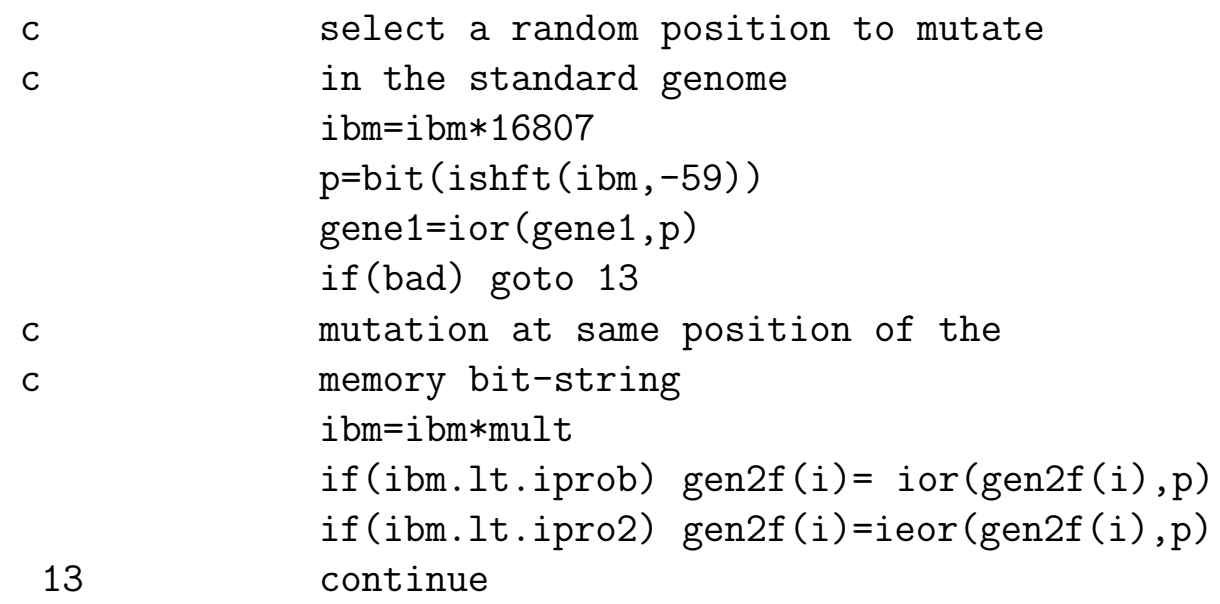

Here, ipro2 corresponds to the probability $q$, iprob to the probability $q(1+q)$, gene1 to the standard genome, and gen2f $(i)$ to the new memory bit-string introduced here. Note that the same random bit $\mathrm{p}$ controls the mutations in the two bit-strings, the array bit(i) $=2^{\mathrm{i}}$ previously defined. (Our random integers ibm used 64 bits and multiplication with mult $=13^{13}$. The logical variable bad is true only during the first 10 percent of the simulation).

Figure 1 shows for $q=0.001$ the effects of the new memory bit-string for good mutations. First we equilibrate the standard model, starting from a random standard genome where on average 16 of the 32 bits are set, and 


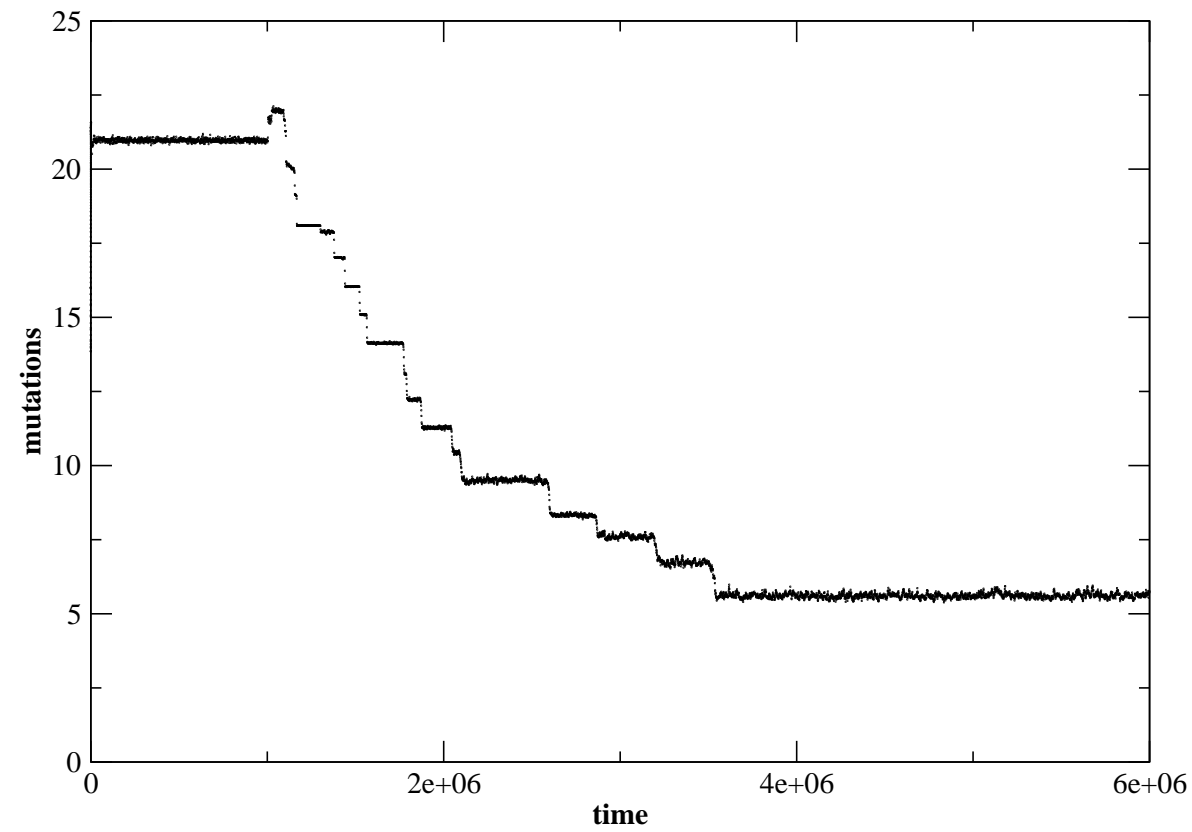

Figure 1: Darwinian evolution of a fitter species over long times at low probability $q=0.001$ with one child per birth; carrying capacity 200,000; actual population increases from about 26,000 to about 37,000 when good mutations on the memory bit-string are switched on at time $10^{6}$.

a completely empty memory genome. After one million iterations, the mutations in the memory genome are switched on, allowing for good mutations with probability $q^{2}=10^{-6}$. From now on the average number of bad mutations in the whole standard bit-string (not counting those made recessive by the memory bit-string) goes down to a much lower value (from 16 to 5). The resulting higher fitness increases the population (not shown). We also see clearly that the improvement of the species proceeds mostly in rapid steps followed by longer intervals of constant number of mutations: "Punctuated equilibrium" as in reality [5]. With $q=0.1$ or 0.01 , this improvement 
proceeds faster (not shown).

Thus, the Penna model allows also an improvement of fitness over long time scales.

\section{Model with mutation-dependent Verhulst factor}

As out pointed in section 1 , in the standard Penna model there is a random time-dependent killing factor $V=N(t) / N_{\max }$, known as the Verhulst factor. At each iteration and for each individual $i$ a random number $r_{i}$ between zero and one is generated and compared with $V$ : if $r_{i} \leq V$, the individual dies independently of its age or genome. Its main purpose is to avoid the exponential increase of the population through a competion for food and space inside an environment that can carry at most $N_{\max }$ individuals. However, in real populations the fitter the animal is, the higher is its probability to win a dispute. So this random killing factor is frequently criticized in the literature 6.

We now introduce a new Verhulst that mostly depends on each individual's current number of mutations, mut, active at it's age:

$$
V_{i}=\frac{N(t)}{N_{\max } \times f_{i}(\mathrm{mut})}, \quad \text { with } f(0)=1 \text { and } f(\text { mut })=\frac{1}{n^{\text {mut }}} .
$$

Now if an individual has not yet accumulated any bad mutation at a given age, it still can be killed by the standard Verhulst probability at that age (since $f(0)=1$ ). However, whenever a new mutation appears, the carrying capacity for that individual is divided by some fixed constant $n$, which increases it's probability to die by the same factor. Also we don't consider anymore a limiting number of bad mutations: the individual dies either by this new Verhulst or because it reaches age 32 .

Figure 2 shows the relative number of individuals with a bit set at a given age, for $n=6$ and for $n=10$. We see that before the minimum reproduction age $R=8$, for $n=6$ all individuals have bits set at ages zero and one, while for $n=10$ the fixation occurs only at age one. In fact, the smaller the value of $n$ is, the higher is the number of fixed bits before $R$. The shape of these curves is similar to those obtained with the standard model [7], although in that case the asexual populations always fix $T-1$ bits before $R$, where 


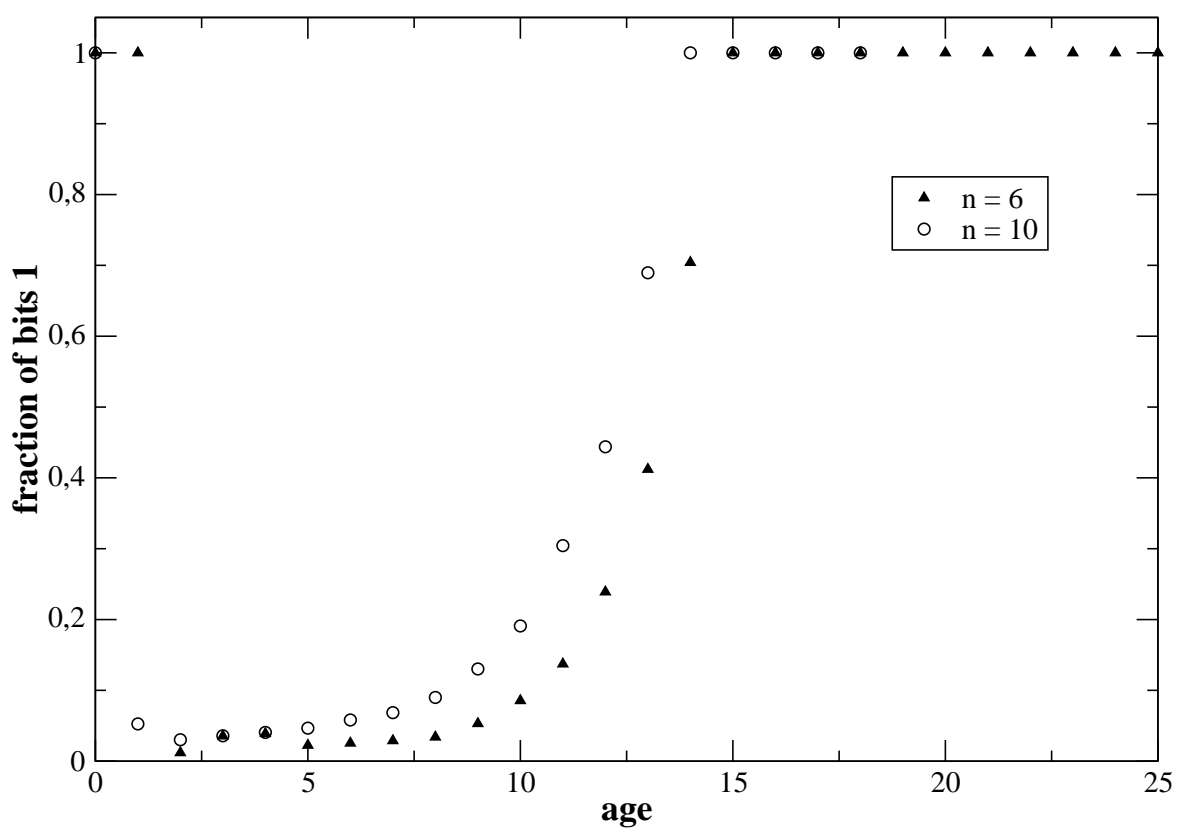

Figure 2: Average fraction of bits set to one per age. Parameters: average population around 120,000 individuals; carrying capacity $=1$ million; one child per birth; minimum reproduction age $=8$; averages taken over the last 10,000 time steps of a total of 1 million time steps. Circles correspond to $n=10$ in the Verhulst factor and triangles to $n=6$.

$T$ is the limit number of allowed diseases. For this reason its longevities are smaller than in the present case, where there is no threshold for bad mutations.

Finally, figure 3 shows the mortality functions, $q(a)$, where $D_{a}$ is the number of deaths at age $a$ :

$$
q(a)=-\ln \left[1-\frac{D_{a}}{N_{a}}\right]
$$




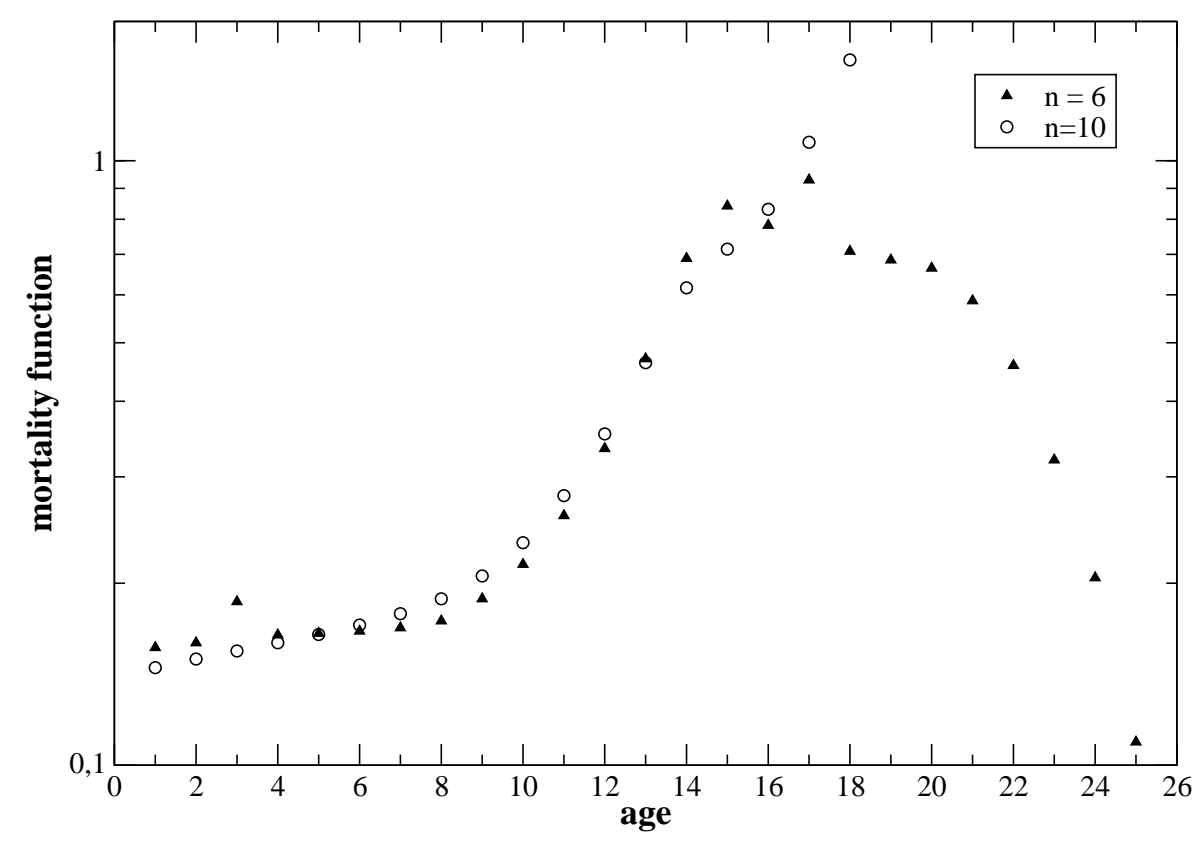

Figure 3: Mortality functions (semi-logarithmic scale) for both cases; same parameters and symbols as before.

Again for the $n=10$ case, the mortality is roughly the same as the usual one, increasing exponentially with increasing age, for ages above the minimum reproduction age (here equal to 8). Such behaviour is known as the Gompertz low of mortality $[8$. For $n=6$, the result changes dramatically and the mortality decelerates at older ages, as observed for drosophilas [9, 10, and may even decrease, as observed for medflies [11]. (For a review on experimental results see [12]). A mortality plateau was also shown to occur within the analytical solution of a slightly modified version of the Penna model [13] where the strict death rule for a fixed number of accumulated mutations is relaxed, although not removed. It was also obtained before with the standard Penna model, but using more complicated strategies as for instance, assum- 
ing a rather unusual antagonistic pleiotropy [14]. Alternative simulations by biologists thus far failed to get this mortality maximum [15].

It is important to say that in the figures presented here we have considered all the results except those for which there was, on average, less than one alive individual with a given age. For this reason, the statistics concerning extremely old individuals is poor. However, such cases correspond to the tails of the curves and don't perturb the reliability of our mortality maximum. For instance, in the $n=6$ case, there is, on average, only one alive individual with age 25 , but there are around 150 with age 18 and 730 with age 16 , where the effect appears.

\section{Conclusions}

Introducing into the Penna bit-string model good mutations or a memory to discount the bad ones (which roughly plays the role of changing a dominant mutation into a recessive one), we obtain an improvement of the population fitness over long time scales. This improvement proceeds mostly in rapid steps followed by longer intervals of constant fitness, meaning that punctuated equilibrium can also be obtained within the Penna model.

We also modify the original model changing the completely random killing Verhulst factor into another one that increases exponentially with the number of active mutations. In this case we don't consider anymore that a given limiting number of mutations kills the individual. Now some individuals can live longer, despite carrying a higher number of genetic diseases, than others which inherited a smaller one. With this modification we reproduce the main features of the original model and also obtain a mortality maximum at old ages.

Acknowledgements: To PMMH at ESPCI for the warm hospitality, to Sorin Tănase-Nicola for helping us with the computer facilities; SMO, PMCO and JSSM thank the Brazilian agencies FAPERJ and CNPq for financial support.

\section{References}

[1] T.J.P. Penna, J. Stat. Phys. 78, 1629 (1995). 
[2] S. Moss de Oliveira, P.M.C. de Oliveira, D. Stauffer: Evolution, Money, War and Computers, Teubner, Stuttgart and Leipzig (1999).

[3] D. Stauffer, page 258 in: Biological Evolution and Statistical Physics, ed. by M. Lässig and A. Valleriani, Springer, Berlin Heidelberg (2002).

[4] D. Stauffer, P.M.C. de Oliveira, S. Moss de Oliveira, T.J.P. Penna, J.S. Sa Martins, An. Acad. Bras. Ci. 73, 15 (2001) (condmat/0011524).

[5] N. Eldredge and S.J. Gould, p.8 in Models in Paleobiology, edited by T.J.M. Schopf, Freeman, San Francisco (1972).

[6] S. Cebrat, Physica A 273, 169 (1999); J.S. Sá Martins and S. Cebrat, Theor. Biosc. 119, 156 (2000); D. Makowiec, Acta Phys. Pol. B31, 1037 (2000); J. Dabkowski, M. Groth and D. Makowiec, Acta Phys. Pol. B31, 1027 (2000).

[7] J.S. Sá Martins and S. Moss de Oliveira, Int.J.Mod.Phys. C 9, 421 (1998).

[8] B. Gompertz, Philos. Trans.R. Soc. London 110, 214 (1825); B. Gompertz, Journal of the Institute of Actuaries 16, 329 (1872).

[9] J.W. Curtsinger, H.H. Fukui, D.R. Townsend and J.W. Vaupel, Science 258, 461 (1992).

[10] D.E.L. Promislow, M. Tatar, A.A. Khazaeli and J.W. Curtsinger, Genetics 143(2), 839 (1996).

[11] J.R. Carey, P. Liedo, D. Orozoco and J.W. Vaupel, Science 258, 457 (1992).

[12] J.W. Vaupel in Between Zeus and the Salmon. The Biodemography of Longevity, ed. K.W. Watcher and C.E. Finch, National Academy Press, Washington D.C. (1997); J.W. Vaupel et al, Science 280, 855 (1998).

[13] J.B. Coe, Y. Mao and M.E. Cates, Phys. Rev. Lett. 89, 288103 (2002).

[14] A.O. Sousa and S. Moss de Oliveira, Physica A 294, 431 (2001); see also S. Moss de Oliveira, P.M.C. de Oliveira and D. Stauffer, Physica A 221, 453 (1995).

[15] L.D. Mueller et al., Experimental Gerontology 38, 373 (2003) 